\title{
Acknowledgment of Ad Hoc Reviewers, 2021
}

The Editor and Associate Editors gratefully acknowledge the following ad hoc reviewers, who reviewed and co-reviewed* manuscripts for the Psychology of Men and Masculinities during the period between August 2020 through August 2021.

Maho Aikawa

Veanne N. Anderson

Jordan Arellanes

Chris Athanasiadis

Jide Bamishigbin

Elyssa C. Berney

Mathieu Bessin

Eve Binks

Ashlee Borgkvist

Lisa Bowleg

Melanie Bozzay*

Evamarie Brock-Midding

Jean-Francois Bureau

Andrew Young Choi

Corey L. Cook

Sam deBoise

Haryana M. Dhillon

Joanna Rachel Doley

W. Justin Dyer

Joshua M. Feinberg

Yanan Feng

Ken Gannon

Kris T. Gebhard

Matthew Genuchi

Zachary T. Gerdes

Anthony Greene

Frederick G. Grieve

Scott Griffiths
Sarah Grogan

Patrick J. Heath

Sarah Hunter

Nur Atikah Mohamed Hussin

Glen Shaun Jankowski

Yahong Jin

Tiana Johnson*

Adam Jowett

Joshua Katz

Mary Keeling

Nikola Komlenac

Karolina Konopka

Vladislav Krivoshchekov

Larkin Lamarche

Suzanne H. Lease

Lucy Longhurst

Stefanie Lopriore

Rory Magrath

Alyssa Marie Glace Maryn

Catherine Massey

Adam M. McCready

Ryan A. McKelley

Katrina McLaughlin

Krista Mincey

Sonia Molloy

Tracy Morison

Christopher D. Nettles
Julia O'Connor

Conor J. O'Dea

James O’Neil

Carlton W. Parks

Kenneth Parnell

Jessica Perrotte

James Ravenhill

Travis Ray

Michael Ray*

Kate Richmond

Damien Ridge

Damien W. Riggs

Martin Robb

Anton Roberts

Lloyd Hawkeye Robertson

Zac Eugene Seidler

Charlene Yvette Senn

Jaclyn A. Siegel

Andrew P. Smiler

Rich Tolman

Beril Türkoğlu

David Vogel

Alan White

David J. Wimer

Shehla A. Yasin

* Denotes a reviewer who co-reviewed under the supervision of a primary reviewer. 